\title{
Reference
}

Firman, J. and Polniaszek (1991). Eight Recommendations for Improving Long-Term Care Insurance. United Seniors Health Cooperative, Washington, DC.

South East London Commissioning Agency,

Camberwell, Lewisham and North Southwark and

West Lambeth Health Authorities,

London SE I 9 RY

\section{Conceptions of Intelligence}

Patrick Rabbitt

Cynthia A. Berg and Robert J. Sternberg, Adults' conceptions of intelligence across the adult lifespan. Psychology and Aging, 7, (1992), $22 I-3 I$.

Robert Sternberg's earlier studies have shown that both expert psychometricians and lay people agree surprisingly well in their definitions of 'intelligence'. Very few follow recent suggestions by Eysenck (1986) and Jensen (1982, 1985) that it must be treated as an abstract global property of the human central nervous system (CNS) which determines the efficiency with which any cognitive task can be performed; that, consequently, the 'biological basis of intelligence' must be discussed in terms of factors that determine the maximum rate at which neural networks can transmit information, and that, if defined in this way, 'intelligence' must inevitably decline as the brain ages and becomes less efficient (Salthouse 1982, 1985, I991). Most people and psychologists define 'intelligence' pragmatically as the ability to solve the problems encountered in everyday life. Zoologists tend towards similar definitions and have difficulty comparing the 'intelligence' of species of different animals with equally successful, albeit intensely specialised, adaptations to diverse ecological niches. In this conceptual framework, intelligence is 'what works best' for an animal or a person. The specialised survival adaptations of academics, aborigines and artists can be regarded as equally valid manifestations of 'intelligence', in the sense that they can all be successful ways of coping with different, complex life demands. In terms of this contextual model of intellectual development, intelligence is defined as 'the mental ability involved in successfully adapting to one's environment'. In the sense that as people age they must adapt to radically altered environments, problems and life-roles, it may be more appropriate to say that their intelligence must certainly qualitatively alter as they grow old but does not necessarily decline. 
This makes Robert Sternberg's latest investigations of the public definitions of intelligence of particular interest to readers of Ageing and Society. Berg and Sternberg ask whether the quality that we define as intelligence, or the general definitions of intelligence on which most are prepared to agree, differ with the ages of the people we take as exemplars. They argue that an exploration of common usage is not trivial because: 'Lay adults' assessments (of intelligence) are important because they serve as the basis for informal assessments of the intelligence of others and self and may impact and be predictive of current and future intellectual performance'. Perhaps they mean that people's views of intelligence may affect the way in which they regard themselves and others, and so may alter their behaviour.

Berg and Sternberg argue that 'intelligence as a concept exists only as a resemblance to the prototype of an intelligent person. That is, degree of intelligence is estimated by the degree to which the individual corresponds to his or her culture's prototype of an exceptionally intelligent person'. It is worth noting that acceptance of this definition lures us well beyond moderate statements by other, equally distinguished, psychometricians who leave some place for the idea that intelligence is at least partly determined by a 'general ability', which is plausibly determined by neurobiology, and so by genetic inheritance, and which may set limits to the efficiency with which new life demands can be met and new roles learned (Spearman, 1904; 1927). An instance is Paul Balthes' balanced remark that, 'with aging... domains of psychological functioning other than performance on intelligence tests gain in relative significance'.

If intelligence is to be defined in terms of socially observed exemplars or prototypes it is important to ask what these may be. Berg and Sternberg asked 152 individuals aged from 17 to 83 years to list behaviours that they regarded as being intelligent. These volunteers produced a list of 2I4 behaviours, which was edited to remove redundancy. A further 140 individuals from the same age range rated each behaviour in this reduced list on a nine point scale for applicability to young ( 30 years), middle-aged ( 50 years), and elderly ( 70 years) people. As Berg and Sternberg expected, there was a marked discrepancy in terms of the attributions of these behaviours to the three different age groups. 'Intelligent behaviours' seen to be characteristic of $3^{\circ}$ year old people correlated with those for $5^{\circ}$ year olds at $r=0.80$, but with those for 70 year olds at only $r=0.16$. Ratings for hypothetical $5^{0}$ and 70 year old's correlated at $r=0.59$. This seems to reflect a marked change in the perceived quality of intelligence between $5^{\circ}$ and 70 years. An elaborate multivariate analysis allowed Berg and Sternberg to suggest that the listed behaviours could be classified into: 


\section{Patrick Rabbitt}

those that exemplified interest and the ability to deal with novelty, those that exemplified everyday competence, and those that exemplified verbal competence. The first were thought to be most characteristic of 30 year olds, the second of 50 year olds, and the third of 70 year olds.

Berg and Sternberg comment that beliefs about the nature of intelligence and about differences in its characteristic manifestations in different age groups are consistent with the idea that intelligence as a property is 'multidimensional and plastic'. Most interestingly, although different qualities of intelligence were seen a characteristic of different times of life, neither the lists of qualities nor their attributions to different age groups changed with the age of the raters. That is, people of all ages seemed to share the same beliefs about the nature of intelligence and about its characteristic manifestation at different points of the lifespan. Only $3.8 \%$ of adults believed that 'intelligence' is a fixed quality which does not alter with age. Berg and Sternberg claim this is strong popular support for a contextual theory of intelligence.

The idea that as we age our intelligence grows not less but only different is warmly sympathetic to my own generation but, alas, we cannot take much comfort from Berg and Sternberg's paper. They have certainly shown that lay people of a local western subculture distinguish many different examples of intelligent behaviour, and that they consistently and systematically order these as being more or less characteristic of young, middle-aged and elderly adults in their own culture. This ordering does not imply that, if specifically questioned, these people would have supported the view that the general level of implementation of intelligence does not change with age. Rather their ratings clearly imply that they perceive characteristically intelligent behaviours that are accessible to the young as being less accessible to the old. The belief that the characteristic manifestations of intelligence in everyday life change with age neither supports nor excludes belief in an overall change in either the level or the accessible manifestations of intelligence. In other words, it would be agreeable if this study provided evidence against the universality of negative stereotyping, but such reassurance is not available.

Different questions are whether and how this study alters our ideas about the most fruitful ways of investigating changes in intelligence throughout the lifespan. It certainly raises interesting points, but none seem to divert psychometric gerontologists from their current theoretical preoccupations. The first point is that definitions of intelligence in common usage are pragmatic and empirical rather than abstract. Intelligence is seen as the apt and elegant deployment of knowledge 
about the world, or the display of long-learned skills such as language. Equally important, however, is the second point that even with this overall pragmatic bias there is room for the definition of intelligence to include a quality of behaviour, such as 'interest' or 'the ability to deal with novelty', which is different from, and cannot be expressed in, any particular learned performance. There is also the third point that this more abstract manifestation of intelligence emphasises a quality, not embodied in any particular acquired skill or knowledge, but rather a prerequisite for acquiring a high level of practical competence in any one of a number of different areas, which is seen as most characteristic of young people. Apparently popular usage endorses both Horn and Cattell's (1967) distinction between fluid and crystalised abilities, and their suggestion that cognitive changes in ageing is a progress from the former to the latter. To this extent, the new information does not invalidate the search for a biological basis of intelligence which might allow us to link changes in the ageing brain to alterations of competence.

What this line of research does is highlight the fact that lay people are as sophisticated in their appraisal of intelligence as most experts; that they quite rightly interpret intelligence as the practical manifestation of strikingly appropriate behaviour, and that they also are enlightened in the sense that they understand that different kinds of behaviour may be appropriate at different points in a person's life cycle. We should not allow our enjoyment of this triumph of common sense to be spoiled by logical or methodological quibbles. For example, that any other outcome than support for an 'exemplar' theory of intelligence would have been very unlikely because Berg and Sternberg specifically asked their respondents to generate and rate only instances of intelligent behaviours. For over one hundred years the progress of psychometrics has depended on a willing suspension of disbelief in the inconvenience of tautology. In these hard times, when threats to full academic employment grow alarmingly, if would be churlish not to continue to make this small effort on behalf of esteemed colleagues.

\section{References}

Eysenck, H. J. 1986. The theory of intelligence and the psychophysiology of cognition. In R. J. Sternberg (ed.), Advances in the Psychology of Human Intelligence. Vol. 3, pp 1-34, Erlbaum, Hillsdale, New Jersey.

Horn, J. L. and Cattell, R. B. 1967 . Age differences in fluid and crystalised intelligence. Acta Psychologica, 26, 107-129.

Jensen, A. R. 1982. The chronometry of intelligence. In R. J. Sternberg (ed.), Advances in the Study of Human Intelligence, Vol. I, Erlbaum, Hillsdale, New Jersey. 


\section{Patrick Rabbitt}

Jensen, A. R. 1985. The nature of the black-white difference on various psychometric tests: Spearman's hypothesis. The Behavioural and Brain Sciences, 8, 193-21 19.

Salthouse, T. A. 1982. Adult Cognition: An Experimental Psychology of Human Aging, Springer-Verlag, New York.

Salthouse, T. A. I 985. A Theory of Cognitive Aging, North Holland, Amsterdam.

Salthouse, T. A. 1991. Theoretical Perspectives on Cognitive Aging. Erlbaum, Hillsdale, New Jersey.

Spearman, C. r904. General intelligence objectively determined and measured. American fournal of Psychology, 15, 20 I-293.

Spearman, C. 1927. The Abilities of Man, Macmillan, London. 